\title{
Gluon Transport Equation in the Small Angle Approximation and the Onset of Bose-Einstein Condensation
}

\author{
Jean-Paul Blaizot ${ }^{\mathrm{a}}$, Jinfeng Liao ${ }^{\mathrm{b}, \mathrm{d}}$, Larry McLerran ${ }^{\mathrm{c}, \mathrm{d}, \mathrm{e}}$ \\ a Institut de Physique Théorique, CNRS/URA 2306, CEA Saclay, F-91191 Gif-sur-Yvette, France \\ ${ }^{b}$ Physics Dept. E CEEM, Indiana University, 2401 N Milo B. Sampson Lane, Bloomington, IN 47408, USA \\ ${ }^{c}$ Physics Dept., Bldg. 510A, Brookhaven National Laboratory, Upton, NY-11973, USA \\ ${ }^{d}$ RIKEN BNL Research Center, Bldg. 510A, Brookhaven National Laboratory, Upton, NY 11973, USA \\ ${ }^{e}$ Physics Department, China Central Normal University, Wuhan, China
}

\begin{abstract}
To understand the evolution of a dense system of gluons, such as those produced in the early stages of ultra-relativistic heavy ion collisions, is an important and challenging problem. We describe the approach to thermal equilibrium using the small angle approximation for gluon scattering in a Boltzmann equation that includes the effects of Bose statistics. The role of Bose statistical factors in amplifying the rapid growth of the population of the soft modes is essential. With these factors properly taken into account, one finds that elastic scattering alone provides an efficient mechanism for populating soft modes, and in fact leads to rapid infrared local thermalization. Furthermore, recent developments suggest that high initial overpopulation plays a key role and may lead to dynamical Bose-Einstein condensation. The kinetics of condensation is an interesting problem in itself. By solving the transport equation for initial conditions with a large enough initial phase-space density the equilibrium state contains a Bose condensate, and we present numerical evidence that such over-occupied systems reach the onset of Bose-Einstein condensation in a finite time. It is also found that the approach to condensation is characterized by a scaling behavior. Finally we discuss a number of extensions of the present study.
\end{abstract}

Keywords: heavy ion collision, quark-gluon plasma, glasma, thermalization, Bose-Einstein condensation

\section{Introduction}

To understand the evolution of a dense system of gluons, such as those produced in the early stages of ultrarelativistic heavy ion collisions, is an important and challenging problem. In such collisions there are strong evidences for the emergence of hydrodynamic expansion on a rather short time scale. Different approaches have been developed to understand exactly how that happens and which mechanism(s) may drive the approach to equilibrium in such a system (see e.g. recent reviews in [1, 2, 3]).

It has been argued recently [4] that the gluonic systems created in the early stages of nucleus-nucleus collisions contain more gluons than can be accommodated by a Bose-Einstein equilibrium distribution. More precisely, the dimensionless number $n / \epsilon^{3 / 4}$, with $n$ the number density and $\epsilon$ the energy density, exceeds typically the maximal value allowed by a thermally equilibrated Bose distribution function. We shall refer to such systems as "over-occupied". For initial conditions where all modes are occupied uniformly up to the saturation scale $Q_{s}$, with occupation factor of order $1 / \alpha_{s}$, the over-occupation parameter is $n / \epsilon^{3 / 4} \sim 1 / \alpha_{s}^{1 / 4}$. It can therefore be large when the coupling constant $\alpha_{s}$ is small. Under such conditions, if the kinetics of the approach to equilibrium is dominated by processes that conserve the number of particles, a Bose condensate will develop as the system evolves towards equilibrium. While the ultimate thermal state is dictated by conservation laws and the occurrence of a condensate can be inferred from 

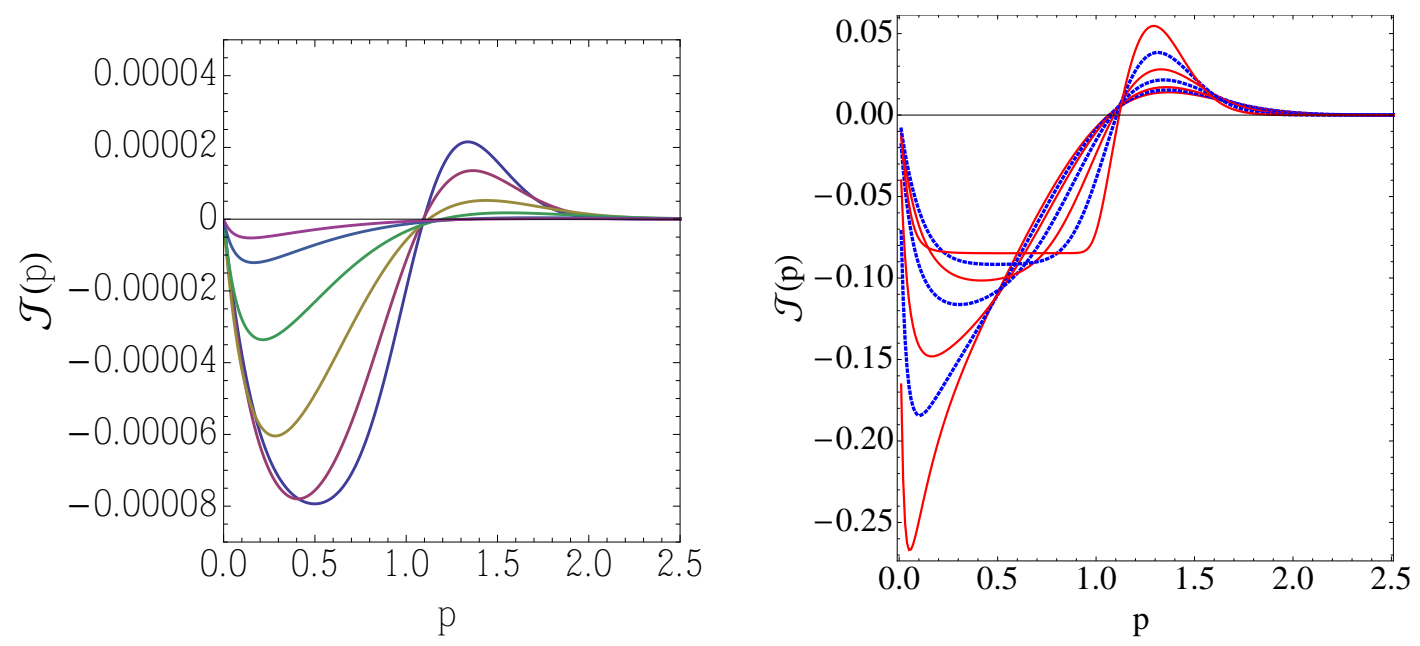

Figure 1. The current $\mathcal{J}(\vec{p})$ at different time moments for underpopulated (left) and overpopulated (right) initial conditions.

initial overpopulation, the dynamical evolution for the onset of such a condensate is an important and interesting question that needs to be understood. In this contribution, we report our study of this phenomenon using a Boltzmann equation for gluons in the small angle scattering limit [5].

\section{Gluon transport equation in the small angle approximation}

In the transport framework, we describe the system of gluons that is produced in an ultra-relativistic heavy ion collision in terms of a distribution function in phase-space, $f(\vec{x}, \vec{p})=\frac{(2 \pi)^{3}}{2\left(N_{c}^{2}-1\right)} \frac{d N}{d^{3} \vec{x} d^{3} \vec{p}}$, where $N$ denotes the total number of gluons in the system. In other words, $f$ denotes the number of gluons of a given spin and color in the phase-space element $d^{3} \vec{x} d^{3} \vec{p} /(2 \pi)^{3}$. We assume that $N$ remains constant during the evolution, that is, we ignore in this work the effect of inelastic, number changing, processes. We also assume that the phase-space densities of different spins and colors are equal, that is, $f$ is independent of spin and color. The time evolution of this distribution function is governed by the kinetic equation that accounts for the gluon-gluon elastic scatterings and that includes the effect of Bose statistics (sometimes referred to as the Boltzmann-Nordheim equation). Under the assumption that small angle scatterings dominate [6], one can in fact reduce the transport equation to a Fokker-Planck equation describing diffusion in momentum space. This allows writing the collision integral as the divergence of a current $\mathcal{J}(\vec{p})$ in momentum space,

$$
\mathcal{D}_{t} f=-\vec{\nabla} \cdot \mathcal{J}=-\frac{\partial \mathcal{J}_{i}}{\partial p_{i}}, \quad \mathcal{J}(\vec{p})=-2 \pi^{2} \alpha^{2} \xi\left[I_{a} \vec{\nabla} f(\vec{p})+\frac{\vec{p}}{p} I_{b} f(\vec{p})[1+f(\vec{p})]\right],
$$

where $\xi=18 \mathcal{L} / \pi$ with $\mathcal{L}=\int_{q_{\min }}^{q_{\max }} d q / q$ the familiar Coulomb logarithm to be regulated by typical hard scale at the ultraviolet and by Debye screening mass at the infrared. The $I_{a}$ and $I_{b}$ are the following integrals

$$
I_{a} \equiv \int \frac{d^{3} p}{(2 \pi)^{3}} f(\vec{p})(1+f(\vec{p})), \quad I_{b} \equiv \int \frac{d^{3} p}{(2 \pi)^{3}} \frac{2 f(\vec{p})}{p} .
$$

The integral $I_{a}$ plays the role of a diffusion constant (to within numerical constants and the Coulomb logarithm, this is the proportionality coefficient between the current and the gradient of $f$ ), while $I_{b}$ is proportional to the squared Debye screening mass, $m_{D}^{2}=2 g^{2} N_{c} I_{b}$. Note that the fixed point solution of the above equation, i.e. the equilibrium distribution function, is the Bose-Einstein distribution $f_{e q}=\frac{1}{\mathrm{e}^{(p-\mu) / T}-1}$. This can be seen from the fact that for an equilibrium distribution function $f_{e q}(p)$, the integrals $I_{a}$ and $I_{b}$ are simply related: $I_{a}=T I_{b}$. It is also straightforward to show that the above collision kernel conserves both energy and particle number.

Given the above kinetic equation, one can then study the system evolution for given initial condition. A particularly simple distribution is that inspired by the color glass (CGC) picture [4]: $f(p)=f_{0} \theta\left(1-p / Q_{s}\right)$ with $Q_{s}$ the saturation scale. By examining the energy and particle number densities of this initial condition, it is easy to see that for $f_{0}<f_{0}^{c} \approx 0.154$ the system is underpopulated while for $f_{0}>f_{0}^{c}$ it is overpopulated [5]. 

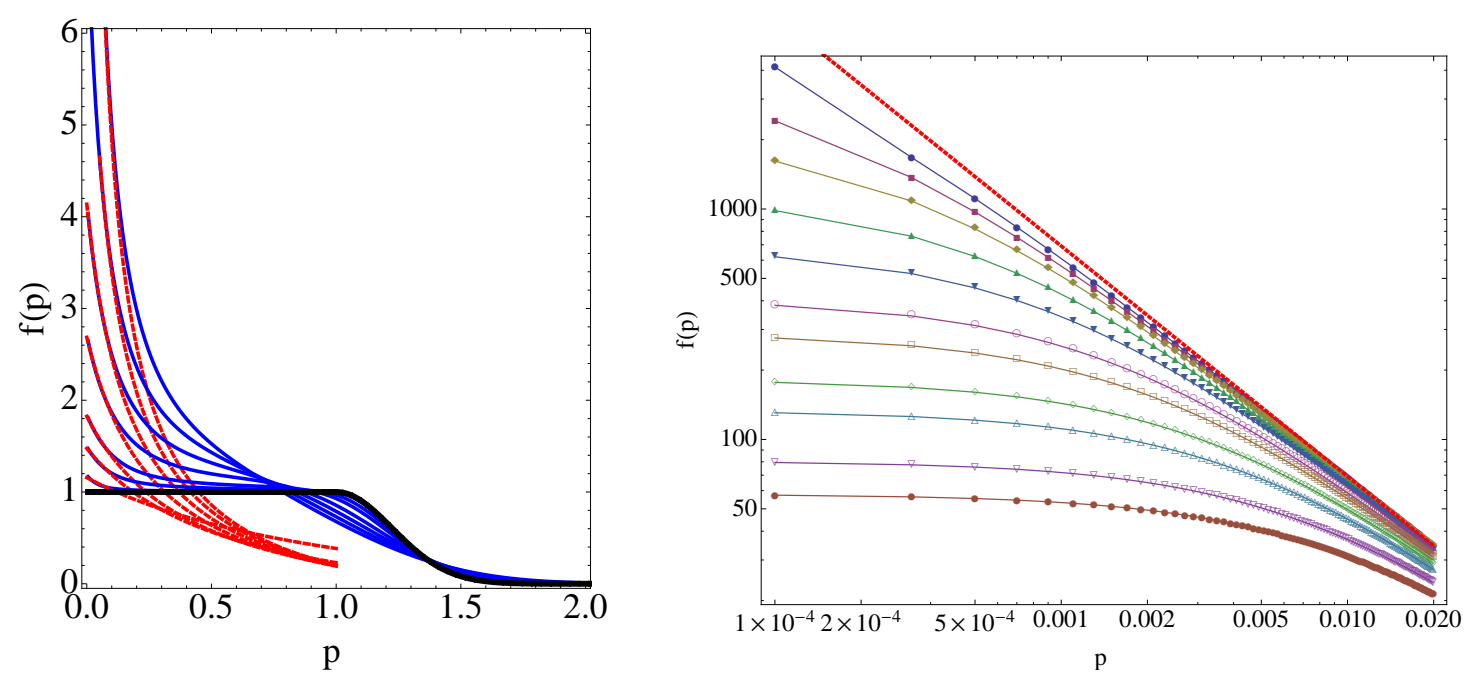

Figure 2. (color online) (left) Time evolution of the distribution $f(p)$ (blue curves) with red dashed curves representing a classical thermal form $T^{*} /\left(p-\mu^{*}\right)$; (right) Evolution from early (lower curves) to later (upper curves) time in deep infrared regime with initial condition $f_{0}=1$.

\section{From overpopulation to the onset of Bose-Einstein condensation}

By numerically solving Eq.(1) with given initial condition, one can study the time evolution of the distribution in great details. One universal feature of such evolution is the occurrence of two cascades: the energy cascade toward the ultraviolet (as a positive current at large momentum) and the particle cascade toward the infrared (as a negative current at small momentum). This is evident from Fig.1: the currents take such a characteristic form for both underpopulated (left, with $f_{0}=0.1$ ) and overpopulated (right, with $f_{0}=1$ ) cases.

An important consequence of the IR cascade is the rapid growth of occupation at very soft momenta, for which the Bose enhancement factor $(1+f)$ for the final state plays an essential role. This further results in an almost immediate IR thermalization, i.e. the distribution in the IR regime quickly evolves into a classical thermal form $f \rightarrow T^{*} /\left(p-\mu^{*}\right)$ with $T^{*}, \mu^{*}$ being the IR local "temperature" and (negative) "chemical potential". This can be seen in Fig.2 (left).

It is then natural to ask how the IR parameters $T^{*}, \mu^{*}$ evolve with time. This however depends on initial conditions. In the underpopulated case, the thermal form will extend from IR to UV and these local parameters will approach the global thermal values with time, as shown in [5]. In the overpopulated case, however, they evolve in a completely different manner. The infrared occupation will keep increasing and driving the local (negative) chemical potential $\mu^{*}$ to vanish in a finite time. This behavior can be seen in Fig.2 (right): in the deep infrared, there is a plateau-like structure $f \sim T^{*} /\left|\mu^{*}\right|$ for $p \ll\left|\mu^{*}\right|$ and a $f \sim 1 / p$ structure for $\left|\mu^{*}\right| \ll p$; with $\left|\mu^{*}\right|$ approaching zero, the plateau shrinks while the $1 / p$ behavior extends more and more toward $p=0$; eventually when $\left|\mu^{*}\right|$ vanishes the IR distribution becomes $1 / p$ (the uppermost, dashed curve). This leads to the emergence of singularity at $p=0$ at a finite time $\tau_{c}$, and marks the dynamical, out-of-equilibrium onset of Bose-Einstein condensation.

We've found this onset process to be quite generic with various (overpopulated) initial conditions. In Fig.3 (left) the time dependence $\mu^{*}(\tau)$ is shown for different $f_{0}$ values. In all cases, the approach of $\mu^{*}$ to zero can be described by a critical scaling behavior for $\tau \rightarrow \tau_{c}$ (where $\tau_{c}$ is the onset time):

$$
\left|\mu^{*}\right|=C\left(\tau_{c}-\tau\right)^{\eta}, \quad \eta \simeq 1
$$

The onset time as a function of initial occupation $f_{0}$ is shown in Fig.3 (right).

\section{Summary}

In summary, we have studied the evolution of a dense gluon system (such as those produced in the early stages of ultra-relativistic heavy ion collisions), by deriving and solving a gluon transport equation with elastic scatterings in the small angle approximation. The Bose statistical factors are found to play an essential role in amplifying scatterings 

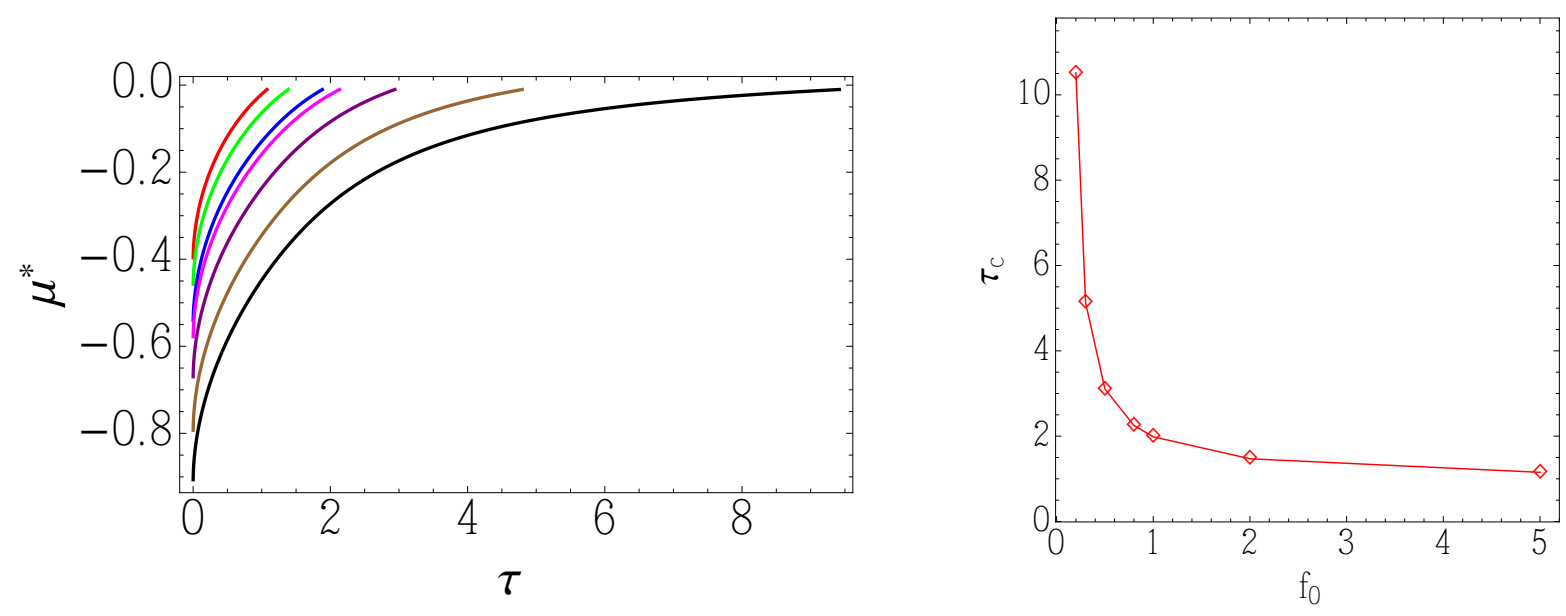

Figure 3. (left) The time dependence $\mu^{*}(\tau)$ for various values of $f_{0}>f_{0}^{c}$; (right) The onset time $\tau_{c}$ versus initial occupation $f_{0}$.

and rapidly populating the soft modes. As a result the infrared regime quickly evolves into a classical thermal form, characterized by a local temperature and chemical potential. Given an overpopulated initial condition, the equilibrium state of the system contains a Bose condensate, and we have presented numerical evidence that such over-occupied systems reach the onset of Bose-Einstein condensation in a finite time. This is achieved by a continuous growth of infrared occupation that drives the local chemical potential $\mu^{*}$ toward zero and the approach to condensation is characterized by a scaling behavior with a universal exponent.

The dynamical evolution from overpopulation to the onset of Bose-Einstein condensation that we've reported here appears to be robust and universal. Extensions to include e.g. medium screening, inelastic processes, quarks, cascade without small angle approximation, etc have been done $[7,8,9,10]$, and similar results have been found. There also seem to be evidences for the link between overpopulation and condensation from classical statistical simulations [11, 12, 13]. Finally let us mention that electromagnetic emissions may provide access to information about the system evolution at the very earliest stages, as recently discussed in e.g. [14, 15].

\section{Acknowledgements}

The research of JPB is supported by the European Research Council under the Advanced Investigator Grant ERCAD-267258. JL is supported by the NSF under Grant No. PHY-1352368. The research of LM is supported under DOE Contract No. DE-AC02-98CH10886. JL also thanks RIKEN BNL Research Center for partial support.

\section{References}

[1] J. Berges, J. -P. Blaizot and F. Gelis, J. Phys. G 39, 085115 (2012).

[2] F. Gelis, Int. J. Mod. Phys. A 28, 1330001 (2013). F. Gelis, arXiv:1312.5497 [hep-ph].

[3] X. -G. Huang and J. Liao, Int. J. Mod. Phys. E 23, 1430003 (2014).

[4] J. -P. Blaizot, F. Gelis, J. -F. Liao, L. McLerran and R. Venugopalan, Nucl. Phys. A 873, 68 (2012).

[5] J. -P. Blaizot, J. Liao and L. McLerran, Nucl. Phys. A 920, 58 (2013).

[6] A. H. Mueller, Nucl. Phys. B 572, 227 (2000); Phys. Lett. B 475, 220 (2000).

[7] X. -G. Huang and J. Liao, arXiv:1303.7214 [nucl-th].

[8] J. -P. Blaizot, B. Wu and L. Yan, arXiv:1402.5049 [hep-ph].

[9] J. -P. Blaizot, Y. Jiang and J. Liao, in final preparation.

[10] F. Scardina, D. Perricone, S. Plumari, M. Ruggieri and V. Greco, arXiv:1408.1313 [nucl-th]. M. Ruggieri, F. Scardina, S. Plumari and V. Greco, arXiv:1312.6060 [nucl-th].

[11] T. Epelbaum and F. Gelis, Phys. Rev. Lett. 111, 232301 (2013); Nucl. Phys. A 872, 210 (2011).

[12] J. Berges and D. Sexty, Phys. Rev. Lett. 108, 161601 (2012); J. Berges, S. Schlichting and D. Sexty, Phys. Rev. D 86, 074006 (2012).

[13] J. Berges, K. Boguslavski, S. Schlichting and R. Venugopalan, Phys. Rev. D 89, 074011 (2014); Phys. Rev. D 89, 114007 (2014); JHEP 1405, $054(2014)$

[14] M. Chiu, T. K. Hemmick, V. Khachatryan, A. Leonidov, J. Liao and L. McLerran, Nucl. Phys. A 900, 16 (2013).

[15] C. Klein-Boesing and L. McLerran, Phys. Lett. B 734, 282 (2014). L. McLerran and B. Schenke, arXiv:1403.7462 [hep-ph]. 